\title{
Influence of Additives to Bovine Bone Material in Osseous Regeneration of Mandibular Defect: An Animal Study using DXA
}

\author{
${ }^{1}$ Mohammad Ali Saghiri, ${ }^{2}$ Jafar Orangi, ${ }^{3}$ Nader Tanideh, ${ }^{4}$ Armen Asatourian, ${ }^{5}$ Franklin Garcia-Godoy, ${ }^{6}$ Nader Sheibani
}

\begin{abstract}
Introduction: Increasing bone quality and quantity in the areas with insufficient bone volume is a major concern among scientists. Ideal bone substitute materials should have osteogenicity, osteoconductivity, and osteoinductivity. Clinoptilolite offers bovine deorganified crystalline bone materials, the advantage of being very similar to human bone with regard to its pore morphology and crystalline structure. This study evaluated the effect of adding Clinoptilolite to Bio-Oss on the osseous regeneration and bone healing process using serial dual-energy X-ray absorptiometry (DXA).
\end{abstract}

Materials and methods: A total of 64 rabbits were anesthetized and a bone defect was created on both semi-mandibles. The rabbits were divided into four equal groups: $\mathrm{A}$ (Bio-Oss ${ }^{\circledR}$ ); $\mathrm{B}$ (Bio-Oss ${ }^{\circledR}$ with $2 \%$ Clinoptilolite mixture); C (allograft); and D receiving no treatment. The bone healing response of animals was tested after 2, 14, 30, and 60 days.

Results: Statistical analysis showed significant differences at time intervals before 14 days between allograft and other groups $(p<0.05)$. In all the defects filled with the tested materials, bone formation was observed subjectively. At 30 - and 60 -day intervals, there were no significant differences between allograft and Bio-Oss with $2 \%$ Clinoptilolite group ( $p=0.052$ and $p=0.260$ respectively) although it was significant in 2 - and 14-day intervals.

Conclusion: Clinoptilolite (2\%) can be used to improve the osteoinduction property of bovine deorganified crystalline bone material. Clinoptilolite can be suggested as a potential material added to bone substitute materials due to its porous structure and buffering capacity and adsorption of a number of serum components which aids the osseous regeneration and healing process.

${ }^{1}$ Research Associate, ${ }^{2}$ Visiting Scholar, ${ }^{3}$ Assistant Professor ${ }^{4}$ Clinical Instructor, ${ }^{5,6}$ Professor

${ }^{1,6}$ Department of Ophthalmology and Visual Sciences, University of Wisconsin School of Medicine and Public Health, Madison Wisconsin, USA

${ }^{2-4}$ Sector of Angiogenesis and Regenerative Surgery Dr. H Afsar Lajevardi Cluster, Shiraz, Islamic Republic of Iran

${ }^{5}$ Bioscience Research Center, College of Dentistry, The University of Tennessee Health Science Center, Memphis Tennessee, USA

Corresponding Author: Mohamad Ali Saghiri, Research Associate, Department of Ophthalmology and Visual Sciences University of Wisconsin School of Medicine and Public Health Madison, Wisconsin, USA, e-mail: saghiri@wisc.edu
Keywords: Bio-Oss, Clinoptilolite, Dual-energy X-ray absorptiometry, Osseous regeneration.

How to cite this article: Saghiri MA, Orangi J, Tanideh N, Asatourian A, Garcia-Godoy F, Sheibani N. Influence of Additives to Bovine Bone Material in Osseous Regeneration of Mandibular Defect: An Animal Study using DXA. Int J Experiment Dent Sci 2016;5(2):104-108.

\section{Source of support: Nil}

Conflict of interest: None

\section{INTRODUCTION}

Bone regeneration and augmentation is a prerequisite in the field of implant dentistry for placing root form implants with long-term durability, which includes a valid surgical procedure for increasing bone quality and quantity in the areas with insufficient bone volume. ${ }^{1,2}$ Osteogenicity, osteoconductivity, and osteoinductivity are ideal characteristics of a biomaterial used for stimulating the osseous regeneration ${ }^{1,2}$; further, achieving an ideal material to substitute bone has been a concern among researchers. The integrity characteristics of calcium phosphate-based materials depend on several elements including the chemical constituents and physical properties like the crystal structure and environmental $\mathrm{pH}$ value of the surrounding tissues. ${ }^{3,4}$ Bone substitute materials like bovine deorganified crystalline bone material (Bio-Oss) should be gradually absorbed and replaced by vital bone tissues. ${ }^{5-7}$ Bio-Oss is deproteinized bovine bone xenograft that has small particle size ( $1 \mathrm{~mm}$ average particle size), resulting in significantly high surface area and high calcium release rate $(9.8 \mathrm{mg} / \mathrm{gm})$; further, its rough topography assists in osteoblastic anchorage and proliferation and synthesis of bone matrix on its surface. ${ }^{8-10}$ Bio-Oss has been shown to be similar to the hydroxyapatite (HA) of human bone as they contain a calcium/phosphate proportion that is similar to bone HA. ${ }^{5}$ The use of Bio-Oss in sinus elevation, ridge augmentation, repair of furcation defects, and repair of vertical intrabony defects has been claimed to be successful. ${ }^{11-13}$

Clinoptilolite, belonging to the aluminosilicate materials, is known as a biocompatible material with perfect molecular structure for capturing heavy metal adsorption capacity from the body without removing useful ions 
and minerals and has a significant buffering capacity. ${ }^{14,15}$ Zeolite is a significant growth promoter and transporter of a number of macro- and microchemical elements, such as calcium, potassium, and sodium that can increase the content of macro- $(\mathrm{Ca}, \mathrm{K}, \mathrm{Na})$ and micro-elements and enhance mineral metabolism in the tissues and the organs. $^{16}$

Clinoptilolite offers bovine deorganified crystalline bone materials the advantage of being very similar to human bone with regard to pore morphology, porosity, and crystalline structure. Zeolites have large empty spaces that can accommodate large cations, molecules, and cationic groups. The presence of pores in bone graft biomaterials has been shown to be important for repairing osseous defects, favoring osteoconduction through osseous growth inside the pores. ${ }^{17,18}$ Besides the porous structure and buffering capacity, an added advantage is that clinoptilolite microtopology provides a large hydrophilic surface area that is widely accepted to provide better cellular adhesion for osteoblasts during bone formation and crystalline HA surface that is conducive to faster osteoblast proliferation and differentiation, which are important for stimulating the bone healing process. ${ }^{19,20}$

Bone mass is an important factor in measuring the quality of the bone. ${ }^{21}$ The most common method of measuring bone mass is called dual-energy X-ray absorptiometry (DXA), which is a reliable and noninvasive method. Dual-energy X-ray absorptiometry has been modified for application in small mammals, facilitating the use of animals in longitudinal studies to quantify bone mass changes. In fact, it is an accurate tool for determining bone mineral density (BMD) in animal models. ${ }^{22,23}$

In accordance with this, the present study was an attempt to use trace additive, Clinoptilolite, to amend bone substitute material properties in order to achieve an ideal one. The hypothesis tested was to examine the effect of adding Clinoptilolite to Bio-Oss bone material during bone regeneration and healing process by using DXA measurement.

\section{MATERIALS AND METHODS}

A total of 64 healthy 6-month-old mature male Dutch rabbits ( $n=64)$, weighing $2500 \pm 200 \mathrm{gm}$, were selected. This study was conducted in accordance with the guidelines and approval of ISO 10993-2. ${ }^{24}$ The research protocol was approved by the Research Ethics Committee of Kamal Asgar Research Center (protocol no. KARC/65A2012-21-6).

The rabbits were divided into four experimental groups. Each group was subdivided into four subgroups with four animals in each subgroup based on the defect filled by A (Bio-Oss ${ }^{\circledR}$ - Geistlich Biomaterials, Wolhusen, Switzerland) as clinically available group; B (Bio-Oss ${ }^{\circledR}$ with $2 \%$ Clinoptilolite - Bear River zeolite Co., CA, USA) as the experimental group; $C$ (allograft collected with bone collector system, Aspeo Bone Collector, Anthogyr, France) as golden standard group; D, received no treatment and served as the control group.

Gamma ray was used for sterilization of clinoptilolite before adding to bone substitute material base on ISO 7405. ${ }^{25}$ The gamma process does not create residuals or impart radioactivity in processed products. This part of the study was similar to that of a study by Saghiri et al. ${ }^{26}$ As explained briefly, the rabbits were anesthetized with intramuscular injection of $10 \%$ ketamine (Alfasan, Woerden, the Netherlands) at a dose of $44 \mathrm{mg} / \mathrm{kg}$ and $2 \%$ Xylazine (Bayer, Munich, Germany) at a dose of $8 \mathrm{~mL} / \mathrm{kg}$. Local anesthesia was administered by infiltration of approximately $0.25 \mathrm{~mL}$ of $3 \%$ lidocaine. The hair on the skin around the ventral surface of the mandible and neck regions was shaved and the skin was prepared, followed by aseptic surgery. A 3-cm incision was made on the ventral surface of the mandible to expose mandibular symphysis. A bone defect was drilled into the mandible of each animal using a round carbide bur in a high-speed handpiece under continuous sterile saline solution irrigation. A bone defect with a dimension of $7 \times 1 \times 1 \mathrm{~mm}$ was created on both semi-mandibles.

The bleeding in defect site was controlled and irrigated with normal saline after drying the site; the materials were mixed according to the manufacturers' instructions and directly placed in the osseous defect cavity. In the control group, the bone defects were left to heal naturally without application of any external material. All the other parameters of placement, including mixing, length, and packing, were kept consistent. The incisions were then closed with 3-0 silk sutures; flunixin $(0.15 \mathrm{mg} / \mathrm{kg})$ as an analgesic drug and penicillin (22000 IU/kg) were injected for 3 days. The animals were subjected to the same diet and environmental conditions.

The animals in each group were sacrificed 2, 14, 30, and 60 days after surgery by keeping them in a $70 \%$ carbon dioxide chamber for 5 to 10 minutes. Subsequently, the animals' semi-mandibles were removed and embedded in $10 \%$ buffered formalin. Dual-energy X-ray absorptiometry scans were performed with a Hologic WI bone densitometer with the serial number of \#84107 (Bedford, Massachusetts, USA), which was calibrated daily in accordance with the manufacturer's recommendations. To measure BMD using DXA, the specimens were positioned centrally at the bottom of a cubic thin-walled plastic container filled with water up to a height of $8 \mathrm{~cm}$, as shown in Figures 1A and B. After a semi-mandible was sunk in the container, the device was set for starting the test. The regional high-resolution mode of the small animal scan protocol (scan field 1.0 [width] $\times 1.2$ [height] $\mathrm{cm}^{2}$, a scan 


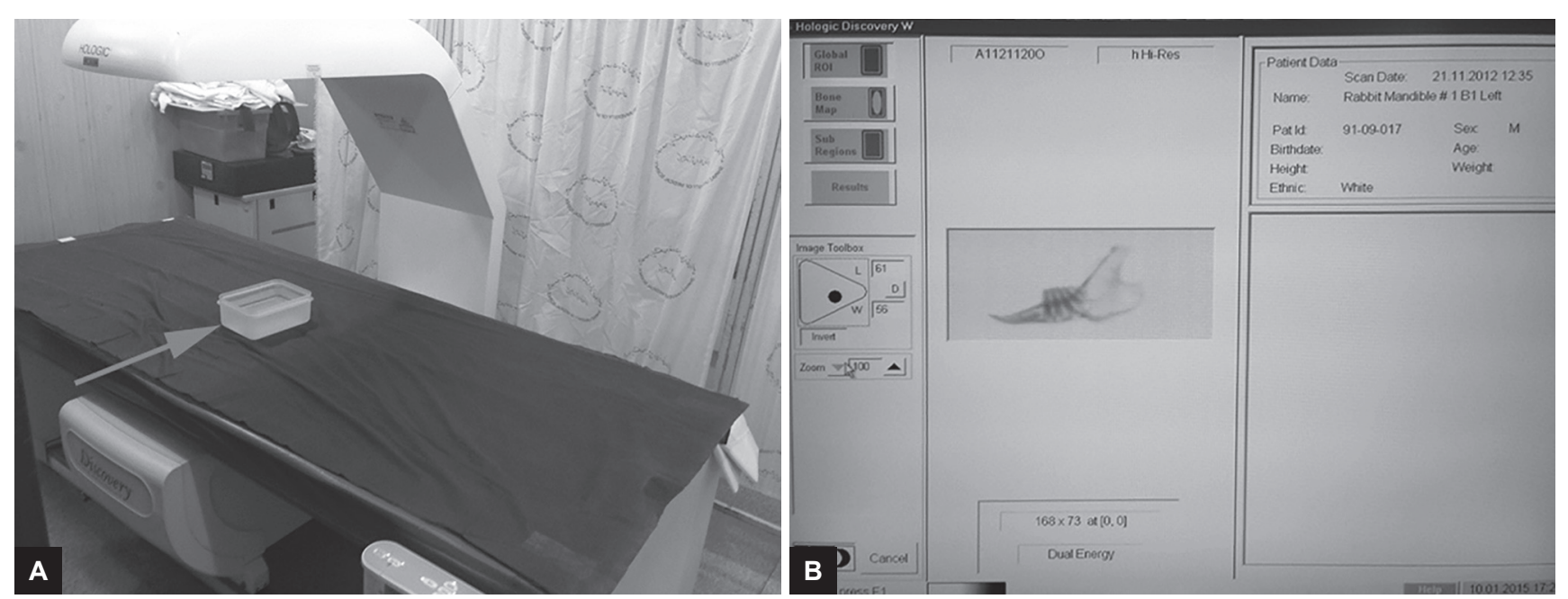

Figs 1A and B: Hologic WI bone densitometer device. A semi-mandible was sunk in the cubic thin-walled plastic filled with water up to a height of $8 \mathrm{~cm}$ and the device was set for starting the test: $(A)$ The red arrow shows position of the plastic container, (B) the device setting applied on software before starting the test

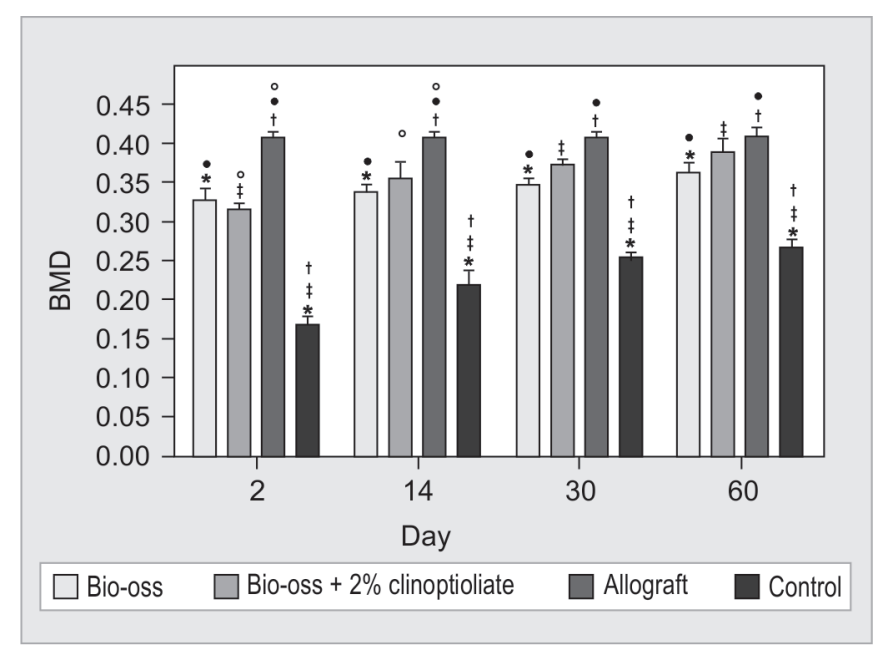

Graph 1: The means and standard deviations of BMD data and the significances for all the experimental groups. Significances between groups at time intervals are specified by symbols

time of 3 minutes) was used. The mandibular bone density of the rabbits was measured and compared in each group. All DXA measurements and analyses were performed by the same operator, who was kept blinded during the analysis. Data were analyzed using two-way analysis of variance, followed by Tukey test, to determine any significant differences between groups at different time intervals. Statistical significance was defined at $p<0.05$.

\section{RESULTS}

The means and standard deviations of BMD results in 2-, 14-, 30-, and 60-day-old specimens are presented in Graph 1 (significant differences are specified with symbols). In all the defects filled with the tested materials, bone formation was observed subjectively. These results demonstrated a consistent increase in BMD values during the time period. There were significant differences between allograft group and other groups at time period before 14 days $(\mathrm{p}<0.05)$. At 30 - and 60-day intervals there were no significant differences between allograft groups and Bio-Oss with $2 \%$ clinoptilolite groups ( $\mathrm{p}=0.052$ and 0.260 respectively). However, there were still significant differences between allograft groups and other groups $(\mathrm{p}<0.05)$.

\section{DISCUSSION}

In the present study, $2 \%$ clinoptilolite was used as an additive to the tested bone substitute material in order to evaluate the effect of this substance on osseous regeneration of the base material in an animal study model. In this study, the methodology was approved previously ${ }^{26}$ and all the animals survived the follow-up period and no complications were noted due to the surgical procedure. In all the defects filled with the test materials, bone formation was observed subjectively.

A pilot study was conducted to ensure that clinoptilolite does not react with the base of bone cement and might not form any new material in accordance with the XRD patterns from different mixture percentages. However, distinguishing the low percentage of clinoptilolite was difficult, and hence, low step size was selected to increase accuracy and intensity of the peaks. In XRD patterns up to $2 \%$ clinoptilolite mixture, new peak was not observed; only the XRD patterns of base material and clinoptilolite could be detected. Whenever more than $2 \%$ clinoptilolite was added to the base material, peaks of clinozoisite, which occurred as a new phase and formed as result of reaction between clinoptilolite and base material, started to intensify and could be detected. Based on the findings of the pilot study, $2 \%$ Clinoptilolite was selected as a reliable amount of mixture to avoid any possible adverse reactions. 
The intake of calcium supplements is highly recommended for bone healing processes. However, the source of calcium plays an important role in the amount of calcium, i.e., assimilated into bone. The presence of pores in bone graft biomaterials has great impact on repairing osseous defects, favoring osteoconduction through osseous growth inside the pores. ${ }^{17,18}$ Clinoptilolite can enhance bone osteoinduction and osteoconduction properties due to its porous structure and hydrophilic surface. According to previous studies, a desirable characteristic of bone substitute materials is their ability to be remodeled, i.e., the biomaterial is absorbed by osteoclasts and is replaced by newly formed bone through osteoblastic activity. ${ }^{27,28}$ Clinoptilolite microtopology provides a large hydrophilic surface area for cell adhesion, which results in faster osteoblast proliferation and differentiation. ${ }^{19,20}$ The results of the current study indicate that adding clinoptilolite as a trace additive increases the hydrophilic surface area and osteoblastic activity, which in turn results in high amounts of newly formed bone and osteoinduction property of bovine deorganified crystalline bone material.

The production of lactic acid under anaerobic conditions in an energy-deficient environment contributes to the acidic microenvironment, resulting in lower $\mathrm{pH}$. This decrease in $\mathrm{pH}$ is expected to influence the surface charge of biomaterials like HA and its derivatives. In healthy tissues, this $\mathrm{pH}$ value is in the range of 7.35 to 7.45. Whenever clinoptilolite is introduced into the body, it buffers the system toward slightly alkaline $\mathrm{pH}$ values (7.35-7.45), which is a perfect buffer for the optimum $\mathrm{pH}$ for the human body. The results of current study showed that the addition of clinoptilolite with its buffering capacity would amend bone healing of Bio-Oss by increasing the $\mathrm{pH}$ of surrounding tissue environment to optimum $\mathrm{pH}$ value.

In all time intervals except the 2-day period, BMD of Bio-Oss group was lower than that of Bio-Oss group with $2 \%$ clinoptilolite, although there was no significant difference between these groups at any other time intervals. This revealed improvements in the effectiveness of the new material to promote new bone formation over time. The results of the current study showed bone healing in Bio-Oss group over time, which is consistent with the findings of other researchers. ${ }^{10,29}$

In all the tested groups, except the allograft group, an increase in BMD data was recorded over time, although the allograft group's response was better than the other groups as expected. The immediate reaction of the allograft group results in the highest BMD among test groups and this is not affected by passing time on placement site.
The negative control group exhibited significant differences from the other test groups at all time intervals. At short intervals (2 and 14 days), Bio-Oss with $2 \%$ clinoptilolite exhibited significant differences from the allograft group, but at 30- and 60-day intervals, there were no significant differences between these two groups. This increase in BMD results of Bio-Oss with $2 \%$ clinoptilolite group might be attributed to an interconnecting pore system introduced by clinoptilolite that allows bony ingrowth and solid integration within the transplantation site and also adsorption of some serum components and influences on ion-exchange kinetics. This will lead to further researches of this material in animals and humans for a deeper understanding of the properties and possibilities that this trace additive might bring into the field of bone healing materials.

\section{CONCLUSION}

The end goal of tissue engineering is to develop products capable of healing diseased or lost tissues and organs. Periodontal regeneration is considered to be organically promising but clinically capricious. ${ }^{30}$

The results of the present study showed that $2 \%$ clinoptilolite can be used to improve the osteoinduction property of bovine deorganified crystalline bone material. This additional substance can be suggested as a potential material added to bone substitute materials due to its porous structure and buffering capacity, and adsorption of a number of serum components which aids the osseous regeneration and healing process.

\section{ACKNOWLEDGMENT}

This project was partially supported from memorial foundation Dr. Hajar Afsar Lajevardi MD, MSc, a legendry Iranian paediatrician (1955-2015). The work in NS lab is supported in part by P30 EY 016665, Retina Research Foundation, and Research to Prevent Blindness unrestricted fund to the department of Ophthalmology and Visual Sciences.

\section{REFERENCES}

1. Habibovic P, de Groot K. Osteoinductive biomaterialsproperties and relevance in bone repair. J Tissue Eng Regen Med 2007 Jan-Feb;1(1):25-32.

2. Tamimi FM, Torres J, Tresguerres I, Clemente C, LópezCabarcos E, Blanco LJ. Bone augmentation in rabbit calvariae: comparative study between Bio-Oss ${ }^{\circledR}$ and a novel $\beta$-TCP/ DCPD granulate. J Clin Periodontol 2006 Dec;33(12):922-928.

3. Radin S, Ducheyne P. Effect of bioactive ceramic composition and structure on in vitro behavior. III. Porous versus dense ceramics. J Biomed Mater Res 1994 Nov;28:1303-1309.

4. Groot K. Effect of porosity and physicochemical properties on the stability, resorption, and strength of calcium phosphate ceramics. Ann NY Acad Sci 1988;523:227-233. 
5. Suzuki T, Hukkanen M, Ohashi R, Yokogawa Y, Nishizawa K, Nagata F, Buttery L, Polak J. Growth and adhesion of osteoblast-like cells derived from neonatal rat calvaria on calcium phosphate ceramics. J Biosci Bioeng 2000;89(1):18-26.

6. Burg KJ, Porter S, Kellam JF. Biomaterial developments for bone tissue engineering. Biomaterials 2000 Dec;21(23):2347-2359.

7. Houser BE, Mellonig JT, Brunsvold MA, Cochran DL, Meffert RM, Alder ME. Clinical evaluation of anorganic bovine bone xenograft with a bioabsorbable collagen barrier in the treatment of molar furcation defects. Int J Periodontics Restorative Dent 2001 Apr;21(2):161-169.

8. Yamada S, Shima N, Kitamura H, Sugito H. Effect of porous xenographic bone graft with collagen barrier membrane on periodontal regeneration. Int J Periodontics Restorative Dent 2002 Aug;22(4):389-397.

9. Wada K, Niimi A, Watanabe K, Sawai T, Ueda M. Maxillary sinus floor augmentation in rabbits: a comparative histologichistomorphometric study between rhBMP-2 and autogenous bone. Int J Periodontics Restorative Dent 2001 Jun;21(3): 252-263.

10. Ekaterina K, Kim Y, Kim J, Kim M-R, Kim SO, Kim S-J. Histomorphometric study on healing of critical sized defect in rat calvaria using three different bovine grafts. J Tissue Eng Regen Med 2012 Oct;9(5):276-281.

11. Camelo M, Nevins ML, Lynch SE, Schenk RK, Simion M, Nevins M. Periodontal regeneration with an autogenous bone-Bio-Oss composite graft and a Bio-Gide membrane. Int J Periodontics Restorative Dent 2001 Apr;21(2):109-120.

12. Smiler DG, Johnson P, Lozada JL, Misch C, Rosenlicht JL, Tatum OH Jr, Wagner JR. Sinus lift grafts and endosseous implants. Treatment of the atrophic posterior maxilla. Dent Clin North Am 1992 Jan;36(1):151-186; discussion 87-88.

13. Abensur D, Wenz B. Sinus grafting with porous bone mineral (Bio-Oss) for implant placement: a 5-year study on 15 patients. Int J Periodontics Restorative Dent 2000 Jun;20(3):245-253.

14. Schainberg A, Özyeğin LS, Kursuoğlu P, Valério P, Goes A, Leite MF. Biocompatibility evaluation of Zeolite compared to bone $\mathrm{HA}$, calcium phosphate $\left(\mathrm{Ca}_{2} \mathrm{PO}_{4}\right)$ and eugenol paste. Key Eng Mater 2005 Apr;284-286:561-564.

15. Li L, Xu W, Grace J. A sustainable approach for acid rock drainage treatment using clinoptilolite. EGU General Assembly Conference Abstracts 2009, Vienna, Austria; 2009. p. 1611.

16. Wu Q, Zhou Y, Wu Y, Wang T. Intestinal development and function of broiler chickens on diets supplemented with clinoptilolite. AJAS 2013 Jul;26(7):987-294.
17. Kalkura SN, Anee T, Ashok M, Betzel C. Investigations on the synthesis and crystallization of hydroxyapatite at low temperature. Bio-Med Mater Eng 2004;14(4):581-592.

18. Werner J, Linner-Krčmar B, Friess W, Greil P. Mechanical properties and in vitro cell compatibility of hydroxyapatite ceramics with graded pore structure. Biomaterials 2002 Nov;23(21):4285-4294.

19. Zanello LP, Zhao B, Hu H, Haddon RC. Bone cell proliferation on carbon nanotubes. Nano Lett 2006 Mar;6(3):562-567.

20. Hunter A, Archer C, Walker P, Blunn GW. Attachment and proliferation of osteoblasts and fibroblasts on biomaterials for orthopaedic use. Biomaterials 1995 Mar;16(4):287-295.

21. Compston J. Bone quality: what is it and how is it measured? Arq Bras Endocrinol Metab 2006 Aug;50(4):579-585.

22. Norris SA, Pettifor JM, Gray DA, Biscardi A, Buffenstein R. Validation and application of dual-energy $X$-ray absorptiometry to measure bone mineral density in rabbit vertebrae. J Clin Densitometry 2000 Spring;3(1):49-55.

23. Kastl S, Sommer T, Klein P, Hohenberger W, Engelke K. Accuracy and precision of bone mineral density and bone mineral content in excised rat humeri using fan beam dualenergy X-ray absorptiometry. Bone 2002 Jan;30(1):243-246.

24. ISO 10993-2:2006 I. Biological evaluation of medical devices Part 2: Animal welfare requirements; 2006.

25. ISO 7405:2008 I. Dentistry - preclinical evaluation of biocompatibility of medical devices used in dentistry - test methods for dental materials; 2008.

26. Saghiri MA, Orangi J, Tanideh N, Janghorban K, Sheibani N. Effect of endodontic cement on bone mineral density using serial dual-energy x-ray absorptiometry. J Endod 2014 May;40(5):648-651.

27. Tamura K, Sato S, Kishida M, Murai M, Ito K. The use of porous $\beta$-tricalcium phosphate blocks with platelet-rich plasma as an onlay bone graft biomaterial. J Periodontol 2007 Feb;78(2): 315-321.

28. Schilling AF, Linhart W, Filke S, Gebauer M, Schinke T, Rueger JM, Amling M. Resorbability of bone substitute biomaterials by human osteoclasts. Biomaterials 2004 Aug;25(18): 3963-3972.

29. Ghanavati F, Gholami GA, Asgari S, Eghbal MJ, Ghanavati F, Rahimi H, Karamlou M, Tamizi M. Bone augmentation potential in rabbit calvaria and ex vivo cytotoxicity of four bone substituting materials. J Periodont Implant Dent 2011;3(1):1-7.

30. Saini R, Saini S, Sharma S. Therapeutics of stem cells in periodontal regeneration. J Nat Sc Biol Med 2011;2:38-42. 